\title{
Guard Band-Based Resource Sharing for Device-to-Device Communications Underlying Cellular Network
}

\author{
Yue Li, Xuejun Sha, Liang Ye, and Xiaojie Fang \\ The Department of Electronics and Information Technology, Harbin Institute of Technology (HIT), Harbin 150001, China \\ Correspondence should be addressed to Xuejun Sha; shaxuejun@hit.edu.cn
}

Received 16 March 2016; Revised 17 June 2016; Accepted 23 June 2016

Academic Editor: Mohammed Nouari

Copyright (C) 2016 Yue Li et al. This is an open access article distributed under the Creative Commons Attribution License, which permits unrestricted use, distribution, and reproduction in any medium, provided the original work is properly cited.

\begin{abstract}
The performance of device-to-device (D2D) communication in a cellular network depends on the resource sharing between D2D links and cellular users. Existing researches on resource sharing mainly focus on power control between the D2D users and cellular users that operate in the same frequency band. However, the D2D outage probability performance is hampered by the cellular interference to D2D links. Therefore, the D2D users may not achieve satisfactory SINR performance when D2D users and cellular users are geographically located in a small area; as a result, the outage probability performance would be significantly degraded. In this paper, we provide a novel resource sharing strategy to mitigate the interference from cellular users to D2D receivers by utilizing the low energy characteristics of signals in the guard band and analyze the D2D outage probability performance mathematically. Both the mathematical analysis and numerical results show that the proposed resource sharing strategy provides $1.2 \mathrm{~dB}$ SNR gain in D2D outage probability performance while guaranteeing the cellular throughputs.
\end{abstract}

\section{Introduction}

Device-to-device communication underlying the cellular network is a promising technology in future wireless networks to improve the resource utilization efficiency and extend coverage areas [1]. However, D2D communication in the cellular spectrum poses a host of challenges to the network. For example, new resource allocation schemes must be designed to mitigate or avoid interference between cellular and D2D links.

Resource allocation methods for D2D users can be categorized into two schemes: the orthogonal resource sharing and the nonorthogonal resource sharing. The former assigns dedicated resources to D2D users, and the latter requires them to share resources with cellular users. The nonorthogonal resource sharing scheme achieves better resource utilization efficiency [2-4]; however, additional manipulations, such as power control, distance limitation, and measures related to mode selection [2-7], are needed to suppress the interference between cellular users and D2D users.

In [2], a resource sharing algorithm is proposed to find the optimal transmission power for D2D communication without degrading the QoS of cellular users. Tang et al. present a series of distributed power control methods to avoid interference and to enhance radio resource utilization in cellular and D2D hybrid networks [3]. Further, Yu et al. employed different resource sharing modes to analyze optimal resource allocation and power control between cellular and D2D connections that share the same resources [4]. In [5-10], the authors considered the similar problems and presented a number of distance-dependent algorithms based on power optimization, uplink reuse allocation, and power management. However, all these solutions hinge on the condition that each D2D link and its paired cellular user operate in the same frequency band, which raises the issue that the D2D receiver may pick up total interference from its paired cellular user. Therefore, the D2D users may not achieve satisfactory SINR (Signal to Interference and Noise Ratio) performance when D2D users and cellular users are geographically located in a small area; as a result, the outage probability performance would be significantly degraded.

In communication systems, to reduce the impact of adjacent channel interference, nearly 10 percent of the system bandwidth at the edge of the allocated bandwidth is reserved 
as the guard band. For example, 3GPP TS 25.101 regulates that the adjacent carrier spacing is $5 \mathrm{MHz}$, but the actual occupied bandwidth is $1+\mu$ times the chip rate in WCDMA system, where $\mu$ is the roll-off factor of the root-raised cosine filter. This leaves the guard band $(5-3.84(1+\mu)) \mathrm{MHz}$. In LTE, when the allocated bandwidth is $20 \mathrm{MHz}$, its actual occupied bandwidth is $18 \mathrm{MHz}$, leaving the guard band $2 \mathrm{MHz}$. Based on the guard band, Chen et al. proposed a novel scheme of utilizing the guard band in LTE uplink, which tried to optimize the overall spectrum efficiency of the two systems adjacently deployed on frequency [11].

In contrast to existing works, this paper considers the low energy characteristics of WCDMA signals in the guard band and presents a novel resource sharing strategy to mitigate cellular user-induced interference at the D2D receiver. In addition, the D2D outage probability performances are discussed mathematically in both flat-fading and frequency-selective channels. Numerical results show that the proposed resource sharing strategy outperforms the conventional strategy.

\section{System Model}

In cellular networks, uplink resource sharing is a common technique, since uplink resources tend to be underutilized in frequency division duplexing (FDD) based cellular networks [12], especially in multimedia services. As a result, uplink resource sharing has been the subject of a number of D2D system designs.

This paper will explore resource sharing problems in one cell, leaving intercell interference out of discussion. Consider a scenario involving a fully loaded cellular network, where $N$ active cellular users occupy $N$ orthogonal channels in a cell, and there are no spare resources. Figure 1 depicts one BS (Base Station) and $N$ orthogonal cellular users, with each user occupying a frequency band indexed by $i=1,2, \ldots, N$. A total of $N-1 \mathrm{D} 2 \mathrm{D}$ pairs exist in this network. The goal of our proposed scheme is to decrease the D2D users' average outage probability with the proposed scheme.

In what follows, we use $\mathrm{DT}_{i}$ and $\mathrm{DR}_{i}$ to denote the $\mathrm{D} 2 \mathrm{D}$ transmitter and receiver, respectively, and the cellular user is denoted by $\mathrm{CU}_{i}$. The following three assumptions are underlying the system model.

Assumption 1. The distance between $\mathrm{DT}_{i}$ and $\mathrm{DR}_{i}$ is assumed to be $\rho_{i}$, and the distances from BS to $\mathrm{CU}_{i}$ and $\mathrm{DT}_{i}$ are denoted by $r_{i}^{c}$ and $r_{i}^{d}$, respectively. For any $\mathrm{CU}_{i}$, the probability density function (PDF) of its distance $r_{i}^{c}$ from BS is $f\left(r_{i}^{c}\right)=2 r_{i}^{c} / R^{2}, r_{i}^{c} \in(0, R][5]$, where $R$ is the cell radius. Moreover, resource sharing is region constrained such that if the $\mathrm{DR}_{i}$ angle is $\theta_{0}$, the cellular user's angle $\theta_{i}$ is uniformly distributed in $\left[\theta_{0}+\pi / 2, \theta_{0}+3 \pi / 2\right)$. And $\Omega$ denotes the set of $\mathrm{CU}_{i}$ locations that meet the region constraint. Since $\rho_{i}$ is much smaller than $r_{i}^{d}$, the distance from BS to $\mathrm{DR}_{i}$ also approaches $r_{i}^{d}$. Assuming that the relative angle between $\mathrm{DR}_{i}$ and $\mathrm{CU}_{i}$ is $\theta$, then the distance between $\mathrm{CU}_{i}$ and $\mathrm{DR}_{i}$ is given by

$$
L_{i}=\sqrt{\left(r_{i}^{c}\right)^{2}+\left(r_{i}^{d}\right)^{2}-2 r_{i}^{c} r_{i}^{d} \cos \theta}
$$

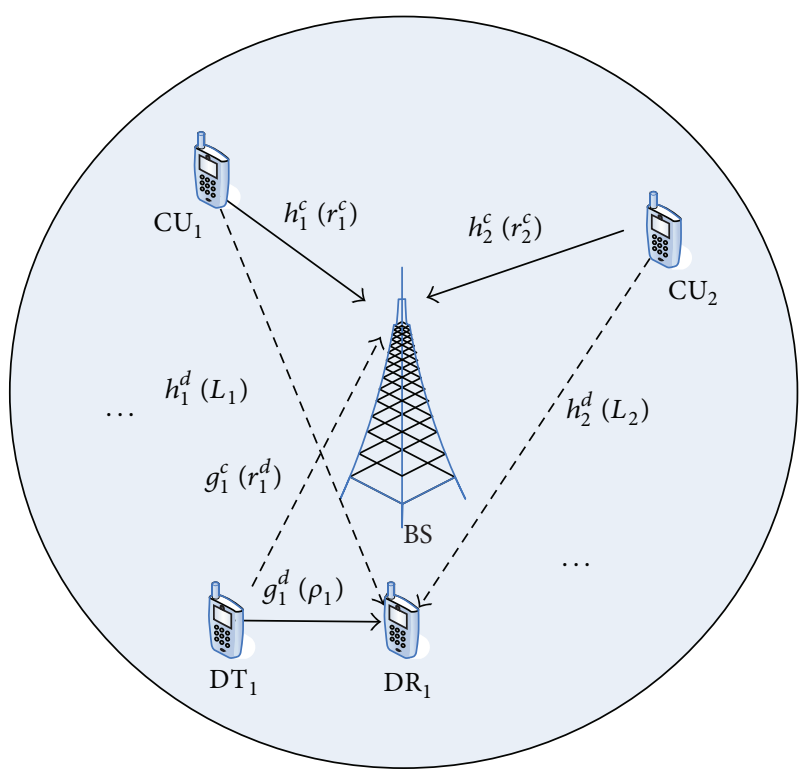

$\longrightarrow$ Signal transmission link

$-\rightarrow$ Interference

FIGURE 1: D2D links sharing uplink resources with cellular users.

Assumption 2. Let $h_{i}^{d}$ and $g_{i}^{d}$ denote the channel from $\mathrm{CU}_{i}$ to $\mathrm{DR}_{i}$ and $\mathrm{DT}_{i}$ to $\mathrm{DR}_{i}$, respectively. Further, the channel from $\mathrm{CU}_{i}$ to $\mathrm{BS}$ is denoted by $h_{i}^{c}$, and the channel from $\mathrm{DT}_{i}$ to BS is given by $g_{i}^{c}$. If channel gain follows independent Rayleigh distribution, then, according to [13], $\left|g_{i}^{d}\right|^{2}$ and $\left|h_{i}^{d}\right|^{2}$ follow independent exponential distribution. In addition, the path loss factor $\alpha$ is set to be 4 [5].

Assumption 3. The system has a guard band between two adjacent channels to suppress interferences between them. The carrier frequency of the cellular user $i$ is denoted as $f_{i}^{c}$ and that of the D2D user $i$ as $f_{i}^{d}$.

\section{Guard Band-Based Resource Sharing}

3.1. Scheme Description. In this part, we will first present the conventional resource sharing technique and then propose a new one to improve the reliability of D2D communications. For convenience, the two strategies are denoted as $\mathscr{M} \mathscr{O} \mathscr{D} \mathscr{E} 1$ and $\mathscr{M} O \mathscr{D} \mathscr{E} 2$, respectively.

In $\mathscr{M} O \mathscr{D} \mathscr{E} 1$, the $\mathrm{D} 2 \mathrm{D}$ user's carrier frequency $f_{i}^{d}$ is equal to its paired cellular user's center frequency $f_{i}^{c}$; that is, $f_{i}^{d}=$ $f_{i}^{c}$. As shown in Figure 2, $B_{L, i}^{d}$ is the lower band bound and $B_{H, i}^{d}$ is the upper band bound of the $\mathrm{DT}_{i} ; B_{L, i}^{c}$ is the lower and $B_{H, i}^{c}$ is the upper band bound of $\mathrm{CU}_{i}$. With $B_{p}$ denoting the passband, $B_{L, i}^{d}=f_{i}^{d}-B_{p} / 2, B_{H, i}^{d}=f_{i}^{d}+B_{p} / 2$, and the same applies to $B_{L, i}^{c}$ and $B_{H, i}^{c}$. Interference received by the D2D link $i$ is caused by $\mathrm{CU}_{i}$, as seen in Figure 2. If the power of the cellular signal $i$ is denoted by $P$, then the cellular interference from $\mathrm{CU}_{i}$ is $P$. 


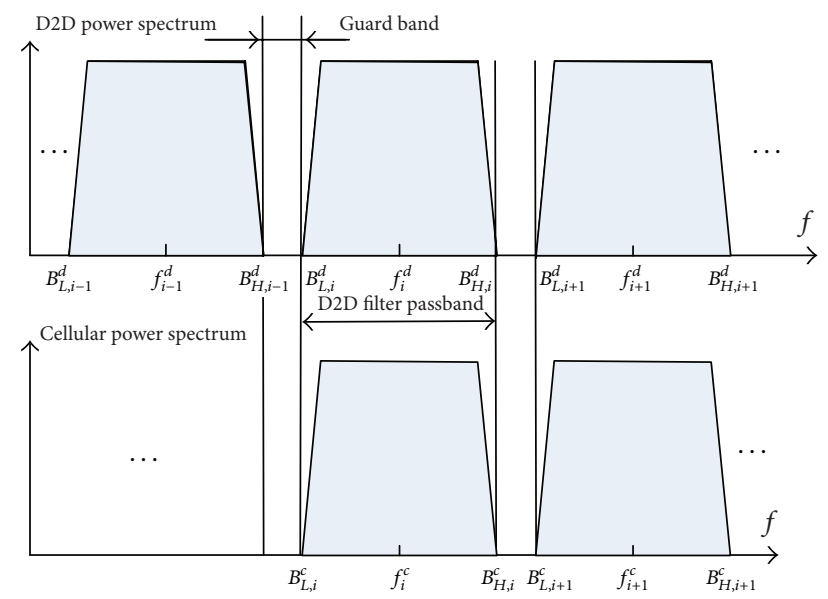

FIGURE 2: Conventional resource sharing strategy $\mathscr{M} \mathscr{O} \mathscr{D} \mathscr{E} 1$.

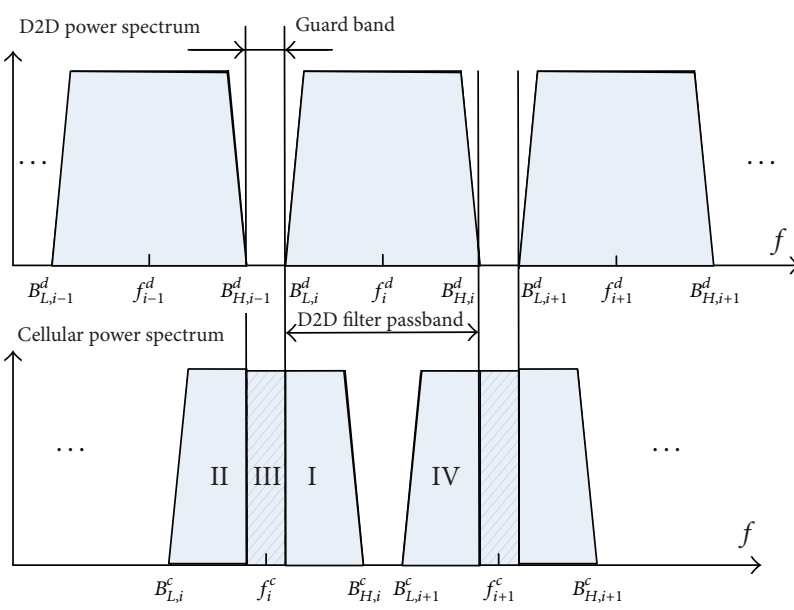

FIGURE 3: Proposed resource sharing strategy $\mathscr{M} \mathscr{O} \mathscr{D} \mathscr{E} 2$.

In $\mathscr{M O D} \mathscr{E} 2, f_{i}^{d}$ is chosen as the center of the guard band between two adjacent cellular channels; that is, $f_{i}^{d}=\left(f_{i}^{c}+\right.$ $\left.f_{i+1}^{c}\right) / 2$. As Figure 3 shows, when the D2D receiver obtains the desired signal with a band-pass filter with passband $\left[B_{L, i}^{d}, B_{H, i}^{d}\right]$, it also picks up cellular interference from $\mathrm{CU}_{i}$ and $\mathrm{CU}_{i+1}$. Denote the power of the interference coming from $\mathrm{CU}_{i}$ as $P_{\mathrm{I}}$ (which means integration over region I in Figure 3) and the power of the interference coming from $\mathrm{CU}_{i+1}$ as $P_{\mathrm{IV}}$. Clearly, the cellular interference is $P_{\mathrm{I}}+P_{\mathrm{IV}}$. Define the interference fraction factor as $\varepsilon, \varepsilon=P_{\mathrm{I}} / P$.

Proposition 4. If a guard band exists between two adjacent channels with center frequencies $f_{i}^{c}$ and $f_{i+1}^{c}$ and the D2D user's center frequency $f_{i}^{d}$ is equal to $\left(f_{i}^{c}+f_{i+1}^{c}\right) / 2$, the defined cellular interference fraction factor $0<\varepsilon<0.5$.

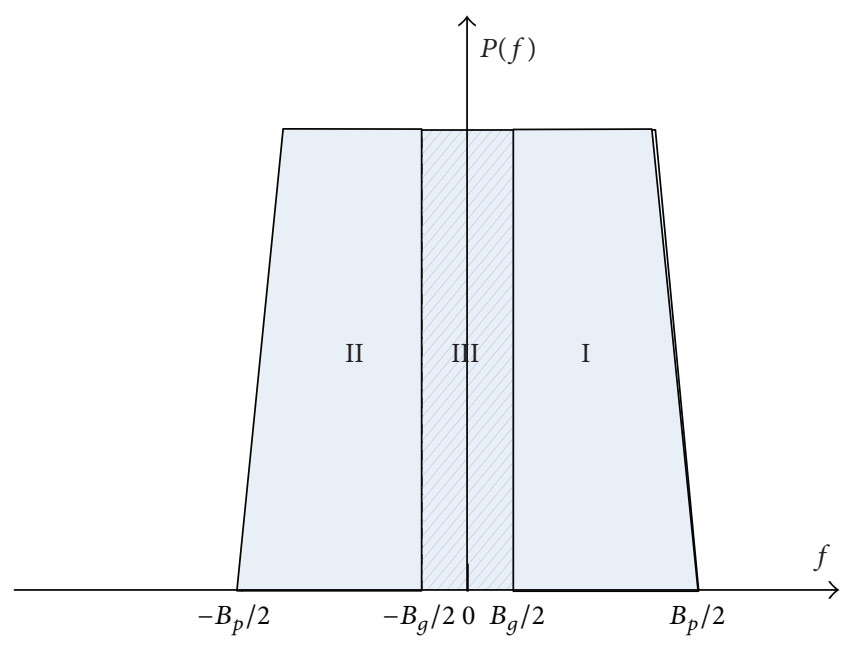

Figure 4: Calculation of $\varepsilon$.

Proof. For convenience, we calculate the parameter $\varepsilon$ in the baseband, as shown in Figure 4. $P(f)$ is the power spectrum, $B_{p}$ is the filter passband, and $B_{g}$ is the guard band. Denote

$$
\begin{gathered}
P_{\mathrm{I}}=\int_{B_{g} / 2}^{B_{p} / 2} P(f) d f \\
P_{\mathrm{II}}=\int_{-B_{p} / 2}^{-B_{g} / 2} P(f) d f \\
P_{\mathrm{III}}=\int_{-B_{g} / 2}^{B_{g} / 2} P(f) d f .
\end{gathered}
$$

Let $P=P_{\mathrm{I}}+P_{\mathrm{II}}+P_{\mathrm{III}}$. Since $P_{\mathrm{I}}=P-P_{\mathrm{II}}-P_{\mathrm{III}}$ and $P_{\mathrm{I}}=P_{\mathrm{II}}, \varepsilon$ can be expressed as

$$
\varepsilon=\frac{P_{\mathrm{I}}}{P}=\frac{1}{2} \frac{\left(P-P_{\mathrm{III}}\right)}{P}=\frac{1}{2}\left(1-\frac{P_{\mathrm{III}}}{P}\right) .
$$

According to (3), it is obvious that $0<P_{\mathrm{III}} / P<1$. As a result, $0<\varepsilon<0.5$.

Equation (3) shows that $\varepsilon$ is affected by the ratio of the interference power in the guard band $P_{\mathrm{III}}$ to the total power $P$. For different CUs, the ratios are identical; that is, the interference fraction factor is also $\varepsilon$ for $\mathrm{CU}_{i+1}$.

3.2. Outage Probability Analysis in a Flat-Fading Channel. Let $x_{j}^{c}(t)$ represent the transmitted signal of $\mathrm{CU}_{j}$ and $x_{i}^{d}(t)$ denote the signal of $\mathrm{DT}_{i}$. In a flat-fading channel, the received $\mathrm{D} 2 \mathrm{D}$ signal at $\mathrm{DR}_{i}$ is

$$
y_{i}^{d}(t)=g_{i}^{d} x_{i}^{d}(t)+\sum_{j} h_{j}^{d} x_{j}^{c}(t)+n_{i}^{d}(t),
$$

where $n_{i}^{d}(t)$ is additive white Gaussian noise with variance $\sigma_{i}^{d}$. Assuming that $p_{j}=E\left|x_{j}^{c}\right|^{2}$ and $q_{i}=E\left|x_{i}^{d}\right|^{2}$, in this paper, a widely used power control scheme for cellular user equipment $\left(\mathrm{CU}_{j}\right)$ is considered, known as the target SNR 
power control scheme (TSPC) [14]. In this scheme, the cellular user's power is selected to reach a fixed SNR (Signal to Noise Ratio) target $\xi$, as shown in (5), where $\sigma_{j}^{c}$ is the variance of the zero-mean Gaussian noise for $\mathrm{CU}_{j}$. In addition, the cellular power $p_{j}$ should satisfy its power constraint $p_{j} \leq$ $P_{c \max }$ :

$$
\xi=\frac{p_{j}\left(r_{j}^{c}\right)^{-\alpha}}{\sigma_{j}^{c}} .
$$

The TSPC scheme is also applied to the D2D link. Two lemmas are utilized to derive the D2D outage probability.

Lemma 5. Let $\left|g_{i}^{d}\right|^{2}$ and $\left|h_{i}^{d}\right|^{2}$ follow independent exponential distribution with unit mean, $x=\left|g_{i}^{d}\right|^{2}$, and $y=a\left|h_{i}^{d}\right|^{2},(a>$ 0 ). Then, the cumulative distribution function (CDF) of $z=$ $x / y$ is

$$
F_{Z}(z)=1-\frac{1}{1+a z}
$$

Proof. The proof is presented in Appendix A.

Lemma 6. Let $\left|g_{i}^{d}\right|^{2},\left|h_{i}^{d}\right|^{2}$, and $\left|h_{i+1}^{d}\right|^{2}$ follow independent exponential distribution with unit mean, and $x=\left|g_{i}^{d}\right|^{2}$ and $y=a\left|h_{i}^{d}\right|^{2}+b\left|h_{i+1}^{d}\right|^{2},(a>0, b>0)$. Then, the CDF of $z=x / y$ is

$$
F_{Z}(z)=1-\frac{1}{(1+a z)(1+b z)} .
$$

Proof. The proof is presented in Appendix B.

The D2D conditional outage probability is given by (8), where $\gamma_{0}$ is the D2D SINR threshold and $\gamma_{i, d}$ is the SINR for $\mathrm{DR}_{i}$, calculated by $(9) . f_{\gamma_{i, d}}(\gamma)$ is the probability density function of $\gamma_{i, d}$. In (9), $P_{s}$ is the power of the desired signal at receiver $\mathrm{DR}_{i}$, and $I_{i}^{c}$ is the cellular interference:

$$
\begin{aligned}
F_{\gamma_{i, d}}\left(\gamma_{0}\right) & =P\left(\gamma_{i, d}<\gamma_{0}\right)=\int_{0}^{\gamma_{0}} f_{\gamma_{i, d}}(\gamma) d \gamma \\
\gamma_{i, d} & =\frac{P_{s}}{\sigma_{i}^{d}+I_{i}^{c}} .
\end{aligned}
$$

The difference between $\mathscr{M O D \mathscr { D } 1}$ and $\mathscr{M O D} \mathscr{E} 2$ lies in the cellular interference $I_{i}^{c}$, so we use a high SNR approximation; that is, $\sigma_{i}^{d} \approx 0$.

3.2.1. The Outage Probability of $M \mathscr{O} \mathscr{D} \mathscr{E} 1$. In $\mathscr{M} \mathscr{O D} \mathscr{E} 1, P_{s}=$ $\left|g_{i}^{d}\right|^{2} \rho_{i}^{-\alpha} q_{i}$ and $I_{i}^{c}=\left|h_{i}^{d}\right|^{2} L_{i}^{-\alpha} p_{i}$; then,

$$
\gamma_{i, d}^{(1)}=\frac{\left|g_{i}^{d}\right|^{2} \rho_{i}^{-\alpha} q_{i}}{\left|h_{i}^{d}\right|^{2} L_{i}^{-\alpha} p_{i}}=\frac{\left|g_{i}^{d}\right|^{2}}{\bar{p}_{i}\left|h_{i}^{d}\right|^{2}},
$$

where $\bar{p}_{i}=p_{i} L_{i}^{-\alpha} / q_{i} \rho_{i}^{-\alpha}$. According to Lemma 5, the conditional outage probability of $\mathscr{M} \mathscr{O} \mathscr{D} \mathscr{E} 1$ can be written as

$$
F_{\gamma_{i, d}}^{(1)}\left(\gamma_{0} \mid r_{i}^{c}, \theta_{i}\right)=1-\frac{1}{1+\gamma_{0} \bar{p}_{i}}
$$

The outage probability of $\mathscr{M O D \mathscr { D }} 1$ can be obtained by averaging over the positions of the cellular user $\mathrm{CU}_{i}$ :

$$
P_{\text {out } 1}=\int_{\Omega} F_{\gamma_{i, d}}^{(1)}\left(\gamma_{0} \mid r_{i}^{c}, \theta_{i}\right) f_{r_{i}^{c}}(r) f_{\theta_{i}}(\theta) d r d \theta \text {. }
$$

3.2.2. The Outage Probability of $M O D \mathscr{D} 2$. In $M O \mathscr{D} \mathscr{E} 2, I_{i}^{c}=$ $\varepsilon \sum_{j=i, i+1}\left|h_{j}^{d}\right|^{2} L_{j}^{-\alpha} p_{j}$; then, the SINR can be expressed as

$$
\begin{aligned}
\gamma_{i, d}^{(2)} & =\frac{\left|g_{i}^{d}\right|^{2} \rho_{i}^{-\alpha} q_{i}}{\varepsilon \sum_{j=i, i+1}\left|h_{j}^{d}\right|^{2} L_{j}^{-\alpha} p_{j}} \\
& =\frac{\left|g_{i}^{d}\right|^{2}}{\varepsilon \bar{p}_{i}\left|h_{i}^{d}\right|^{2}+\varepsilon \bar{p}_{i+1}\left|h_{i+1}^{d}\right|^{2}},
\end{aligned}
$$

where $\bar{p}_{i}=p_{i} L_{i}^{-\alpha} / q_{i} \rho_{i}^{-\alpha}$ and $\bar{p}_{i+1}=p_{i+1} L_{i+1}^{-\alpha} / q_{i} \rho_{i}^{-\alpha}$. The conditional outage probability of $\mathscr{M} \mathscr{O D} \mathscr{E} 2$ can be evaluated with Lemma 6, which is shown by

$$
\begin{aligned}
& F_{\gamma_{i, d}}^{(2)}\left(\gamma_{0} \mid r_{i}^{c}, r_{i+1}^{c}, \theta_{i}, \theta_{i+1}\right) \\
& \quad=1-\frac{1}{\left(1+\gamma_{0} \varepsilon \bar{p}_{i}\right)\left(1+\gamma_{0} \varepsilon \bar{p}_{i+1}\right)} .
\end{aligned}
$$

Similarly, when averaging over the positions of $\mathrm{CU}_{i}$ and $\mathrm{CU}_{i+1}$, the outage probability of $\mathscr{M} \mathscr{O} \mathscr{D} \mathscr{E} 2$ can be obtained:

$$
\begin{gathered}
P_{\text {out } 2}=\int_{\Omega} \int_{\Omega} F_{\gamma_{i, d}}^{(2)}\left(\gamma_{0} \mid r_{i}^{c}, r_{i+1}^{c}, \theta_{i}, \theta_{i+1}\right) f_{r_{i}^{c}, r_{i+1}^{c}}\left(r_{1}, r_{2}\right) \\
\cdot f_{\theta_{i}, \theta_{i+1}}\left(\theta_{1}, \theta_{2}\right) d r_{1} d r_{2} d \theta_{1} d \theta_{2} .
\end{gathered}
$$

3.3. Outage Probability Analysis in a Frequency-Selective Channel. When the system's communication bandwidth is much larger than the coherence bandwidth, the channel has a frequency-selective characteristic. In this case, the channel is modelled as a multipath fading channel, and the received signal is given by

$$
\begin{aligned}
y_{i}^{d}(t)= & \sum_{l=0}^{L-1} \delta_{i, l}^{d} x_{i}^{d}\left(t-\tau_{i, l}\right) \\
& +\sum_{j} \sum_{l=0}^{L-1} \lambda_{j, l}^{d} x_{j}^{c}\left(t-\tau_{j, l}\right)+n_{i}^{d}(t),
\end{aligned}
$$

where $\delta_{i, l}^{d}$ is the channel gain of the $l_{\text {th }}$ path from $\mathrm{DT}_{i}$ to $\mathrm{DR}_{i}$ and $\lambda_{j, l}^{d}$ is the channel gain of the $l_{\text {th }}$ path from $\mathrm{CU}_{j}$ to $\mathrm{DR}_{i}$. Here, $\delta_{i, l}^{d}=g_{i, l}^{d} m_{i, l}^{d}$ and $\lambda_{j, l}^{d}=h_{j, l}^{d} m_{j, l}^{d}$, where $g_{i, l}^{d}$ and $h_{j, l}^{d}$ represent Rayleigh fading and $m_{i, l}^{d}, m_{j, l}^{d}$ represent multipath fading for each path. In contrast to a flat-fading channel, intersymbol interference (ISI) is easily introduced in the multipath environment. Its SINR is extended to

$$
\gamma_{i, d}=\frac{P_{s}}{\sigma_{i}^{d}+I_{i}^{c}+I_{\mathrm{ISI}}^{d}},
$$


where $I_{\text {ISI }}^{d}$ is the intersymbol interference. With a RAKE receiver, multipath components whose delays are less than one chip period can be used to increase the signal energy, thus allowing $P_{s}$ to be written as (18). Let $L_{1}$ be the set of paths whose delays are less than one chip period $T_{c}$; that is, $L_{1}: l \in\left\{\tau_{i, l}<T_{c}\right\}$; then,

$$
P_{s}=\sum_{l \in L_{1}}\left|\delta_{i, l}^{d}\right|^{2} \rho_{i}^{-\alpha} q_{i}
$$

Intersymbol interference originates from multipath signals whose delays exceed one chip period. This set can be defined as $L_{2}: l \in\left\{\tau_{i, l} \geq T_{c}\right\}$ :

$$
I_{\mathrm{ISI}}^{d}=\sum_{l \in L_{2}}\left|\delta_{i, l}^{d}\right|^{2} \rho_{i}^{-\alpha} q_{i}
$$

In $\mathscr{M} \mathscr{O} \mathscr{D} \mathscr{E} 1$, cellular interference $I_{i}^{c}$ can be written as

$$
I_{i}^{c}=\sum_{l=0}^{L-1}\left|\lambda_{i, l}^{d}\right|^{2} L_{i}^{-\alpha} p_{i}
$$

Substitute (18), (19), and (20) into (17); the SINR for $\mathscr{M O D} \mathscr{E} 1$ can be then expressed as

$$
\gamma_{i, d}^{(1)}=\frac{\sum_{l \in L_{1}}\left|\delta_{i, l}^{d}\right|^{2} \rho_{i}^{-\alpha} q_{i}}{\sigma_{i}^{d}+\sum_{l=0}^{L-1}\left|\lambda_{i, l}^{d}\right|^{2} L_{i}^{-\alpha} p_{i}+\sum_{l \in L_{2}}\left|\delta_{i, l}^{d}\right|^{2} \rho_{i}^{-\alpha} q_{i}} .
$$

In $\mathscr{M O D} \mathscr{E} 2$, cellular interference $I_{i}^{c}$ can be written as

$$
I_{i}^{c}=\varepsilon \sum_{l=0}^{L-1}\left|\lambda_{i, l}^{d}\right|^{2} L_{i}^{-\alpha} p_{i}+\varepsilon \sum_{l=0}^{L-1}\left|\lambda_{i+1, l}^{d}\right|^{2} L_{i+1}^{-\alpha} p_{i+1} .
$$

Substitute (18), (19), and (22) into (17); the SINR for $M \mathscr{M O} \mathscr{E} 2$ can be then expressed as

$$
\begin{aligned}
& \gamma_{i, d}^{(2)} \\
& =\frac{\sum_{l \in L_{1}}\left|\delta_{i, l}^{d}\right|^{2} \rho_{i}^{-\alpha} q_{i}}{\sigma_{i}^{d}+\varepsilon \sum_{j=i, i+1} \sum_{l=0}^{L-1}\left|\lambda_{j, l}^{d}\right|^{2} L_{j}^{-\alpha} p_{j}+\sum_{l \in L_{2}}\left|\delta_{i, l}^{d}\right|^{2} \rho_{i}^{-\alpha} q_{i}} .
\end{aligned}
$$

Substitute (21) and (23) into (8), respectively; then, the outage probabilities of $\mathscr{M} O \mathscr{D} \mathscr{E} 1$ and $\mathscr{M} \mathscr{O} \mathscr{D} \mathscr{E} 2$ are obtained. In the following session, we will evaluate the system performance in the frequency-selective channel by simulation.

\section{Performance Comparison}

This section presents a performance comparison between $\mathscr{M O D} \mathscr{E} 1$ and $\mathscr{M O D} \mathscr{E} 2$. Table 1 gives the simulation parameters. In a WCDMA system, the channel spacing is $5 \mathrm{MHz}$, and the transmission pulse shaping filter is a root-raised cosine (RRC) function. The chip duration $T_{c}$ is $260.42 \mathrm{~ns}$ $(1 / 3.84 \mathrm{MHz})$. Assume the roll-off factor $\mu$ to be 0.075 . Since $B_{p}=(1+\mu) / T_{c}$, the signal bandwidth $B_{p}$ is $4.12 \mathrm{MHz}$, making the guard band $\left(B_{q}\right) 0.88 \mathrm{MHz}$. Equation (3) allows us to obtain the interference fraction factor $\varepsilon$, which is 0.3847 .
TABLE 1: Simulation parameters.

\begin{tabular}{lc}
\hline Parameters & Values \\
\hline Cell radius $R$ & $500 \mathrm{~m}$ \\
Maximum cellular power $P_{c \max }$ & $20 \mathrm{dBm}$ \\
Distance between D2D TX and RX $\rho_{i}$ & $\sim U[50 \mathrm{~m}, 100 \mathrm{~m}]$ \\
Distance between D2D TX and BS $r_{i}^{d}$ & $\sim U[250 \mathrm{~m}, 500 \mathrm{~m}]$ \\
Channel spacing & $5 \mathrm{MHz}$ \\
Pulse shaping filter & $\mathrm{RRC}$ \\
Gaussian noise powers $\sigma_{i}^{d}$ and $\sigma_{i}^{c}$ & $-100 \mathrm{dBm}$ \\
D2D SINR threshold $\gamma_{0}$ & $0 \mathrm{~dB}$ \\
\hline
\end{tabular}

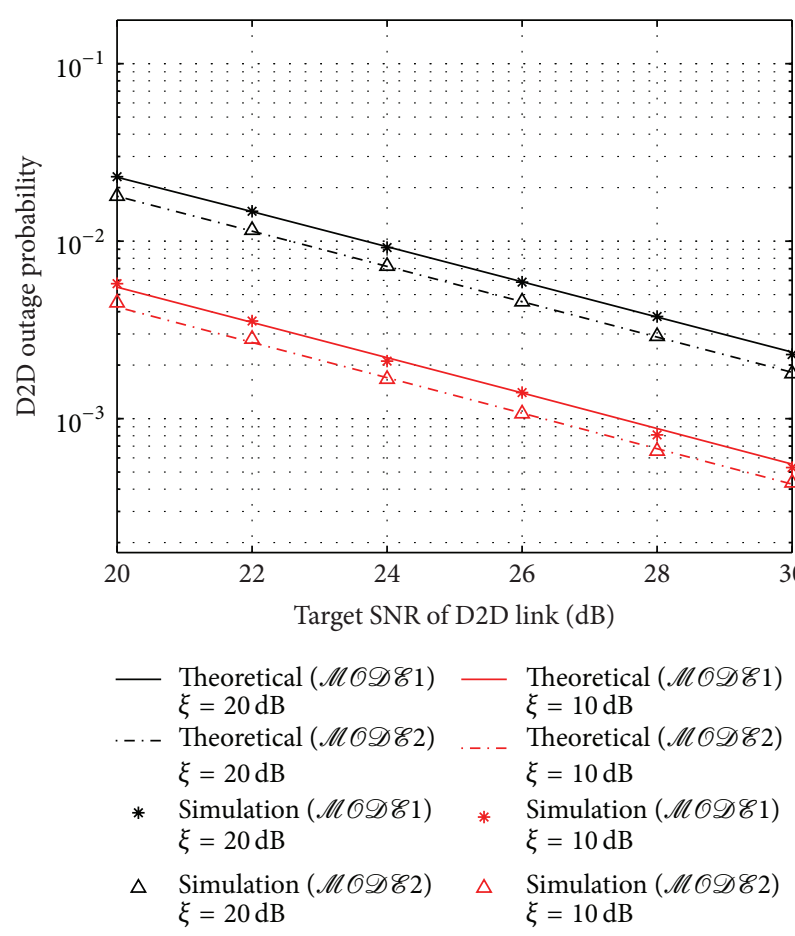

FIGURE 5: D2D outage probability in a flat-fading channel.

4.1. Performance in a Flat-Fading Channel. Figure 5 shows the outage probability performance of D2D communication, indicating how the outage probability changes when the target cellular SNR $\xi$ is set to $10 \mathrm{~dB}$ and $20 \mathrm{~dB}$, respectively. It can be seen that $\mathscr{M} \mathscr{O D} \mathscr{E} 2$ provides better outage probability performance than $\mathscr{M O} \mathscr{D} \mathscr{E} 1$. At the same outage probability level, $\mathscr{M} O \mathscr{D} \mathscr{E} 2$ provides an SNR gain of $1.2 \mathrm{~dB}$, which accords with the theoretical analysis, verifying that our derivation for outage probability in a flat-fading channel is correct.

Figure 6 gives the cellular network's capacity improvement factor with the target cellular SNR $\xi$ set to $5 \mathrm{~dB}, 10 \mathrm{~dB}$, and $20 \mathrm{~dB}$, respectively. Calculated with (26), the capacity improvement factor $\eta$ is averaged over a range of channel states and cellular user positions. In Figure $6, \eta>1$ means that the cellular capacity performance of $\mathscr{M} \mathscr{O} \mathscr{D} \mathscr{E} 2$ is better than that of $\mathscr{M O D} \mathscr{E} 1$. In other words, $\mathscr{M} \mathscr{O} \mathscr{D} \mathscr{E} 2$ 


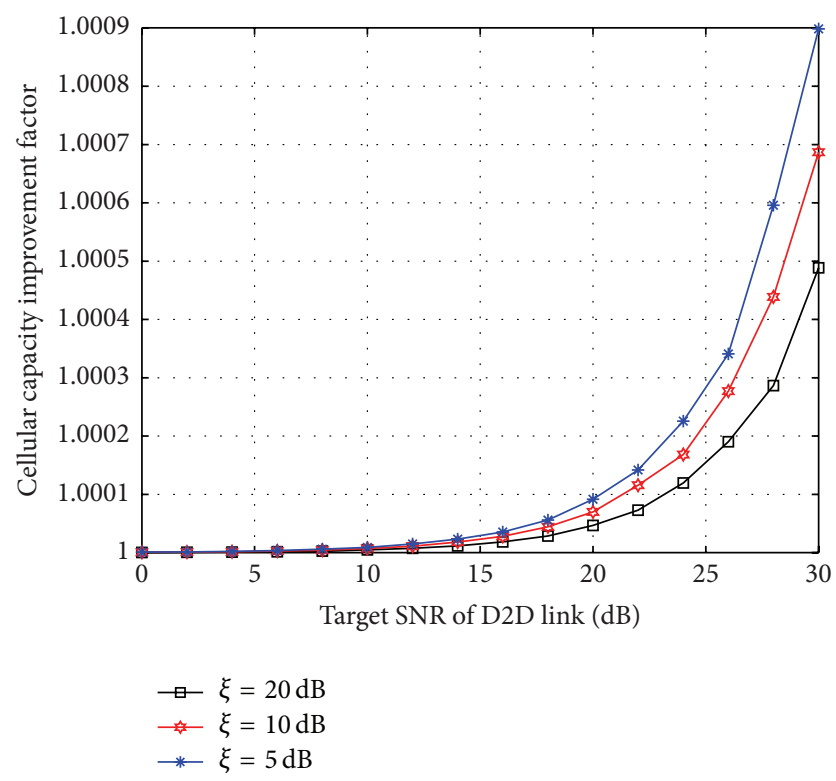

FIGURE 6: Cellular capacity improvement factor in a flat-fading channel.

TABle 2: Ped B channel parameters.

\begin{tabular}{lcc}
\hline Path number & Relative delay & Average power \\
\hline 1 & $0 \mathrm{~ns}$ & $0 \mathrm{~dB}$ \\
2 & $100 \mathrm{~ns}$ & $-3.6 \mathrm{~dB}$ \\
3 & $200 \mathrm{~ns}$ & $-7.2 \mathrm{~dB}$ \\
4 & $300 \mathrm{~ns}$ & $-10.8 \mathrm{~dB}$ \\
5 & $500 \mathrm{~ns}$ & $-18.0 \mathrm{~dB}$ \\
6 & $700 \mathrm{~ns}$ & $-25.2 \mathrm{~dB}$ \\
\hline
\end{tabular}

provides better D2D outage probability performance while guaranteeing the cellular capacity:

$$
\begin{aligned}
R_{i, c}^{(1)} & =\log \left(1+\frac{\left|h_{i}^{c}\right|^{2}\left(r_{i}^{c}\right)^{-\alpha} p_{i}}{\sigma_{i}^{c}+\left|g_{i}^{c}\right|^{2}\left(r_{i}^{d}\right)^{-\alpha} q_{i}}\right) \\
R_{i, c}^{(2)} & =\log \left(1+\frac{\left|h_{i}^{c}\right|^{2}\left(r_{i}^{c}\right)^{-\alpha} p_{i}}{\sigma_{i}^{c}+\varepsilon \sum_{j=i, i-1}\left|g_{j}^{c}\right|^{2}\left(r_{j}^{d}\right)^{-\alpha} q_{j}}\right) \\
\eta & =\frac{R_{i, c}^{(2)}}{R_{i, c}^{(1)}} .
\end{aligned}
$$

4.2. Performance in a Frequency-Selective Channel. The frequency-selective channel is modelled in accordance with the Ped B model defined by ITU. Table 2 presents the channel parameters of this outdoor to indoor pedestrian model. Relative delay refers to a time difference relative to the first path, whereas average power refers to power fading relative to the first path.

Since WCDMA has a chip period of $260.42 \mathrm{~ns}$, the first three paths $(1,2$, and 3$)$ are located in set $L_{1}$ and can be processed by the RAKE receiver. As the other three paths

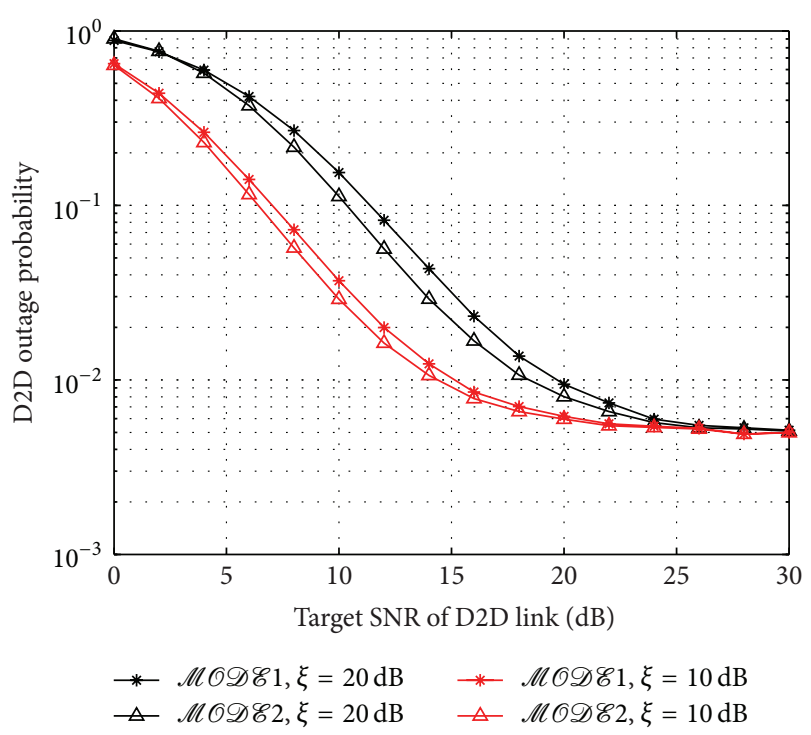

FIGURE 7: D2D outage probability in a frequency-selective channel.

$(4,5$, and 6$)$ are located in set $L_{2}$, they are considered as ISI interference.

Figure 7 shows the outage probability performance in a frequency-selective channel with the target cellular SNR $\xi$ set to 10 and $20 \mathrm{~dB}$, respectively. At low and moderate SNRs, $M O D \mathscr{E} 2$ achieves better outage probability performance than $\mathscr{M} O D \mathscr{E} 1$, with an SNR gain of about $1.2 \mathrm{~dB}$. However, at high SNRs, the outage probabilities of the two modes become equivalent, because, in a high SNR region, the outage probability is mainly affected by $I_{\text {ISI }}^{d}$. The value of $I_{\text {ISI }}^{d}$ is determined by the $\mathrm{D} 2 \mathrm{D}$ transmission power, and it changes with the D2D SNR, making the ISI interferences $I_{\text {ISI }}^{d}$ of the two modes identical.

\section{Conclusion}

This paper investigated resource sharing strategies for D2D communications in a cellular network. In contrast to previous research, we aimed at mitigating cellular interference on the basis of the low energy characteristics of signals in the guard band and proposed a novel resource sharing strategy. Mathematical analysis and simulation results show that, in a flat-fading channel, the proposed strategy could improve the outage probability performance. And in a frequency-selective channel, the advantage of the proposed method was obvious in low and moderate SNR regions.

\section{Appendix}

\section{A. Proof of Lemma 5}

Let $\left|g_{i}^{d}\right|^{2}$ and $\left|h_{i}^{d}\right|^{2}$ follow independent exponential distribution with unit mean, $x=\left|g_{i}^{d}\right|^{2}$, and $y=a\left|h_{i}^{d}\right|^{2},(a>0)$. The PDF of $x$ and $y$ are expressed as $f_{X}(x)=\exp (-x)$ and 
$f_{Y}(y)=(1 / a) \exp (-(1 / a) y)$, respectively. According to [15] (6-43), the PDF of $z=x / y$ is

$$
\begin{aligned}
f_{Z}(z) & =\int_{0}^{\infty} y f_{X Y}(z y, y) d y \\
& =\int_{0}^{\infty} y f_{X}(z y) f_{Y}(y) d y .
\end{aligned}
$$

Substituting $f_{X}(x)$ and $f_{Y}(y)$ into (A.1),

$$
f_{Z}(z)=\frac{1}{a} \int_{0}^{\infty} y \exp \left(-\left(z+\frac{1}{a}\right) y\right) d y .
$$

Using integration by parts, the PDF in (A.2) can be evaluated as

$$
f_{Z}(z)=\frac{1}{a(z+1 / a)^{2}} .
$$

The CDF of variable $Z$ can be expressed as

$$
\begin{aligned}
F_{Z}(z) & =\int_{0}^{z} f_{Z}(s) d s=\int_{0}^{z} \frac{1}{a(s+1 / a)^{2}} d s \\
& =1-\frac{1}{1+a z} .
\end{aligned}
$$

Thus, the desired result (6) is obtained.

\section{B. Proof of Lemma 6}

Let $\left|g_{i}^{d}\right|^{2},\left|h_{i}^{d}\right|^{2}$, and $\left|h_{i+1}^{d}\right|^{2}$ follow independent exponential distribution with unit mean, and $x=\left|g_{i}^{d}\right|^{2}$ and $y=a\left|h_{i}^{d}\right|^{2}+$ $b\left|h_{i+1}^{d}\right|^{2}, \quad(a>0, \quad b>0)$. The PDF of $x$ is expressed as $f_{X}(x)=\exp (-x)$. Let $A=1 / a$ and $B=1 / b$. According to [15] (6-41), the PDF of $y$ is

$$
f_{Y}(y)= \begin{cases}\frac{A B}{B-A}\left(e^{-A y}-e^{-B y}\right) & B \neq A \\ A^{2} y e^{-A y} & B=A .\end{cases}
$$

Since the cellular position is randomly generated, it is more common that $B \neq A$, so we consider the first case. According to (A.1),

$$
\begin{aligned}
& f_{Z}(z)=\int_{0}^{\infty} y e^{-z y} \frac{A B}{B-A}\left(e^{-A y}-e^{-B y}\right) d y \\
& =\frac{A B}{B-A}\left(\int_{0}^{\infty} y e^{-(z+A) y} d y-\int_{0}^{\infty} y e^{-(z+B) y} d y\right) .
\end{aligned}
$$

Applying integration by part to the first and second term of (B.2), respectively, we obtain

$$
f_{Z}(z)=\frac{A B}{B-A}\left(\frac{1}{(z+A)^{2}}-\frac{1}{(z+B)^{2}}\right) .
$$

The CDF of variable $Z$ can be expressed as

$$
\begin{aligned}
F_{Z}(z) & =\int_{0}^{z} \frac{A B}{B-A}\left(\frac{1}{(s+A)^{2}}-\frac{1}{(s+B)^{2}}\right) d s \\
& =1-\frac{A B}{(z+A)(z+B)} .
\end{aligned}
$$

By substituting $A$ and $B$ with $1 / a$ and $1 / b$, respectively, the desired result (7) is obtained.

\section{Competing Interests}

The authors declare that they have no competing interests.

\section{Acknowledgments}

This work was supported by the 973 Program under Grant no. 2013CB329003 and the National Science and Technology Major Project under Grant no. 2012ZX03003011-004.

\section{References}

[1] G. Fodor, E. Dahlman, G. Mildh et al., "Design aspects of network assisted device-to-device communications," IEEE Communications Magazine, vol. 50, no. 3, pp. 170-177, 2012.

[2] J. H. Wang, D. H. Zhu, C. M. Zhao, J. C. F. Li, and M. Lei, "Resource sharing of underlaying device-to-device and uplink cellular communications," IEEE Communications Letters, vol. 17, no. 6, pp. 1148-1151, 2013.

[3] R. Tang, J. Zhao, and H. Qu, "Distributed power control for energy conservation in hybrid cellular network with Device-toDevice communication," China Communications, vol. 11, no. 3 , pp. 27-39, 2014.

[4] C.-H. Yu, K. Doppler, C. B. Ribeiro, and O. Tirkkonen, "Resource sharing optimization for device-to-device communication underlaying cellular networks," IEEE Transactions on Wireless Communications, vol. 10, no. 8, pp. 2752-2763, 2011.

[5] H. Wang and X. Chu, "Distance-constrained resource-sharing criteria for device-to-device communications underlaying cellular networks," Electronics Letters, vol. 48, no. 9, pp. 528-530, 2012.

[6] D. Wu, J. Wang, R. Q. Hu, Y. Cai, and L. Zhou, "Energy-efficient resource sharing for mobile device-to-device multimedia communications," IEEE Transactions on Vehicular Technology, vol. 63, no. 5, pp. 2093-2103, 2014.

[7] Y. Q. Chai, Q. H. Du, and P. Y. Ren, "Partial timefrequency resource allocation for device-to-device communications underlaying cellular networks," in Proceedings of the IEEE International Conference on Communications (ICC '13), pp. 6055-6059, Budapest, Hungary, June 2013.

[8] D. Feng, L. Lu, Y.-W. Yi, G. Y. Li, G. Feng, and S. Li, "Device-todevice communications underlaying cellular networks," IEEE Transactions on Communications, vol. 61, no. 8, pp. 3541-3551, 2013.

[9] Z. Liu, T. Peng, H. Chen, and W. Wang, "Optimal D2D user allocation over multi-bands under heterogeneous networks," in Proceedings of the IEEE Global Communications Conference (GLOBECOM '12), pp. 1339-1344, Anaheim, Calif, USA, December 2012.

[10] S. W. Xiang, T. Peng, Z. Y. Liu, and W. Wang, "A distancedependent mode selection algorithm in heterogeneous D2D and IMT-Advanced network," in Proceedings of the IEEE Globecom Workshops (GC Wkshps '12), pp. 416-420, Anaheim, Calif, USA, December 2012.

[11] W. Q. Chen, G. F. Cui, J. Duan, W. D. Wang, and Y. H. Zhang, "Research on utilizing guard band in LTE uplink co-existence based on uncoordinated deployment scenario," in Proceedings of the IET International Conference on Communication Technology and Application (ICCTA '11), pp. 441-446, Beijing, China, October 2011. 
[12] M. Zulhasnine, C. Huang, and A. Srinivasan, "Efficient resource allocation for device-to-device communication underlaying LTE network," in Proceedings of the 6th Annual IEEE International Conference on Wireless and Mobile Computing, Networking and Communications (WiMob '10), pp. 368-375, Niagara Falls, Canada, October 2010.

[13] J. G. Proakis, Digital Communications, Publishing House of Electronics Industry, 2007.

[14] P. Janis, V. Koivunen, C. Ribeiro, J. Korhonen, K. Doppler, and K. Hugl, "Interference-aware resource allocation for device-todevice radio underlaying cellular networks," in Proceedings of the IEEE 69th Vehicular Technology Conference (VTC Spring '09), pp. 1-5, Barcelona, Spain, April 2009.

[15] A. Papoulis and S. U. Pillai, Probability, Random Variables and Stochastic Process, McGraw-Hill, New York, NY, USA, 4th edition, 2002. 


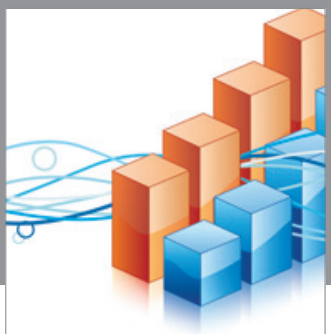

Advances in

Operations Research

vatem alat4

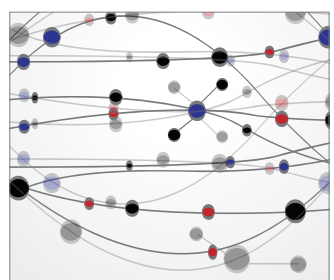

\section{The Scientific} World Journal
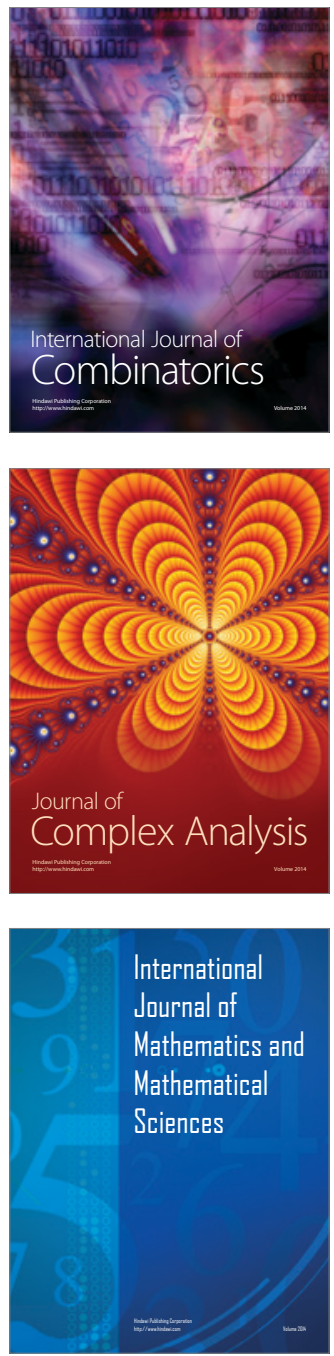
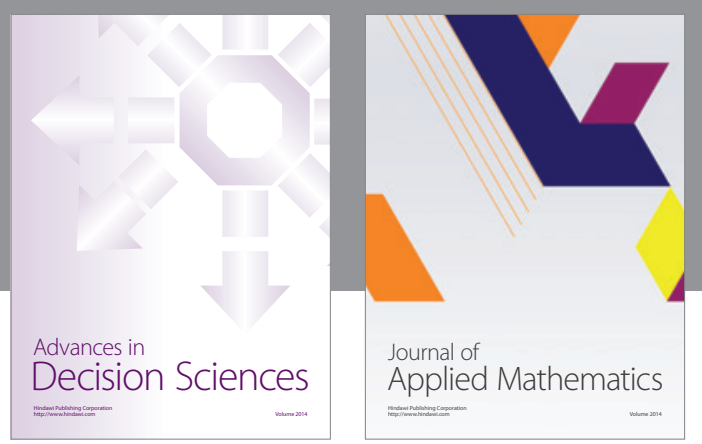

Algebra

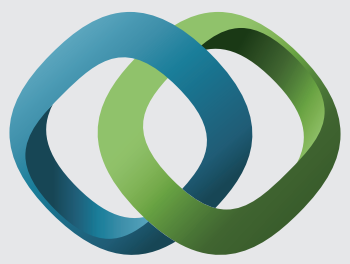

\section{Hindawi}

Submit your manuscripts at

http://www.hindawi.com
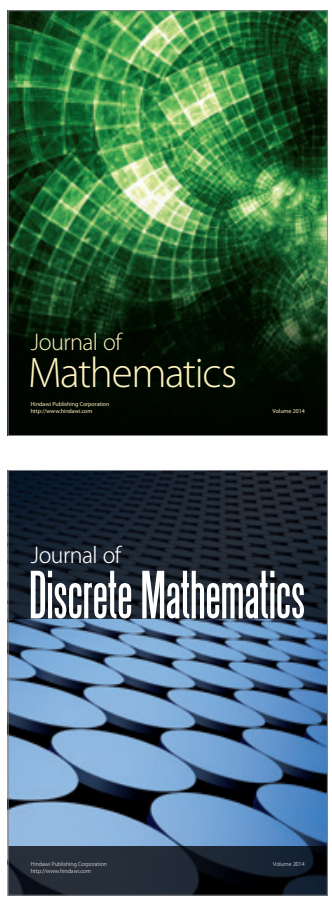

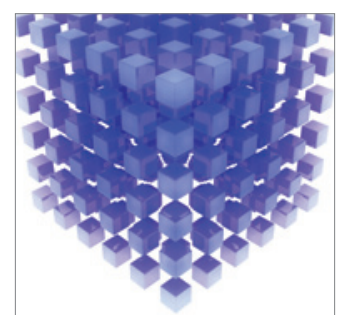

Mathematical Problems in Engineering
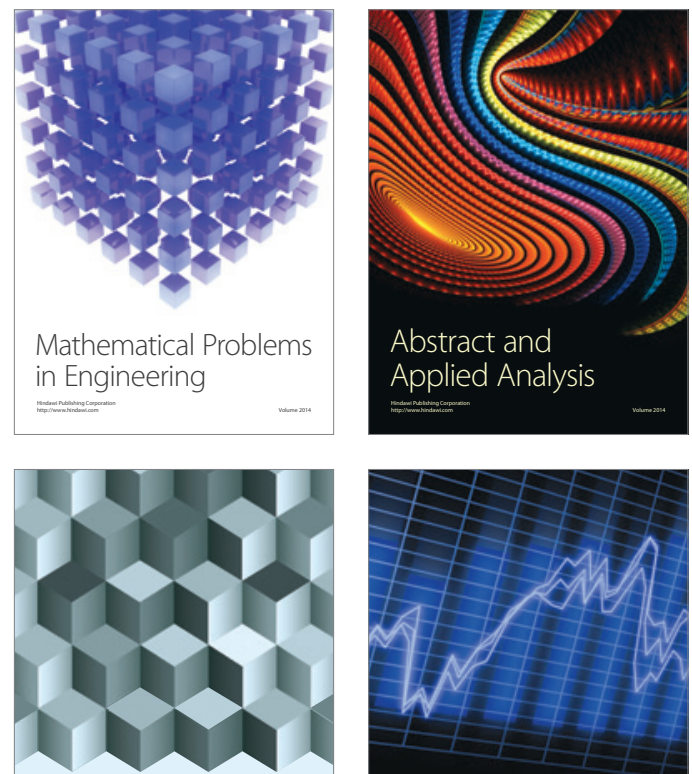

Journal of

Function Spaces

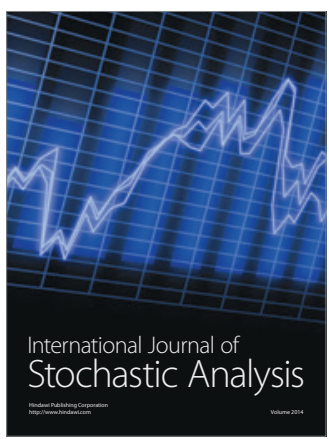

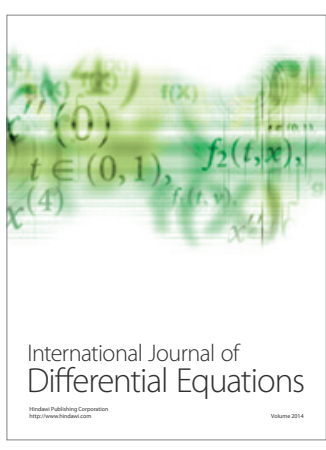
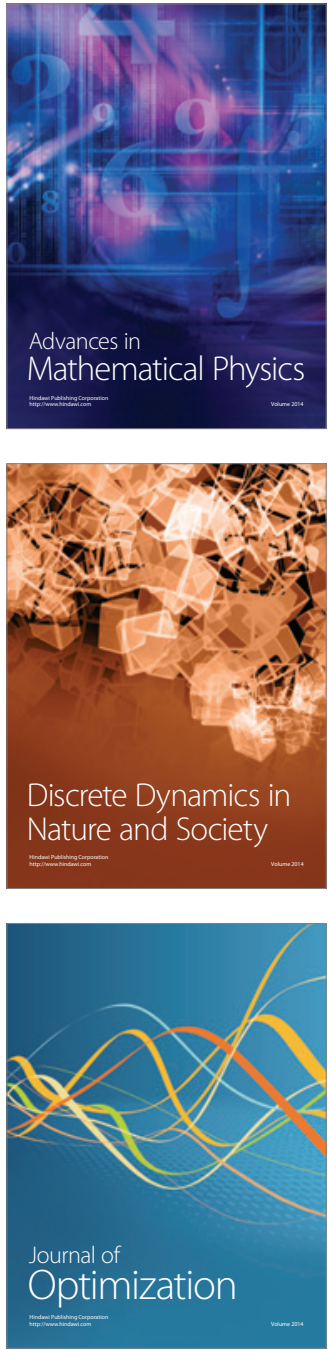